\title{
Differences in Factors Predicting Outcome and Mortality after Mechanical Thrombectomy for Acute Large Vessel Stroke Between Patients over and Under 70 Years of Age: A Five Year Retrospective Study
}

\author{
Timothy Derkhoong Woo, Anand Sastry, Vincent Leung, Christine Roffe and Sanjeev Nayak* \\ University Hospital of North Staffordshire, Newcastle Road, United Kingdom
}

Received: March 03, 2015; Accepted: June 29, 2015; Published: July 02, 2015

*Corresponding author: Sanjeev Nayak, University Hospital of North Staffordshire, Newcastle Road, Stoke-on-Trent, ST4 6QG, United Kingdom, Tel: 01782 679023; E-mail: sanjeev.nayak@uhns.nhs.uk

\begin{abstract}
Aim: To assess the differences in risk factors between different age groups after mechanical thrombectomy (MT) for ischaemic stroke, including recanalization time and functional and radiological scoring.

Methods: Patients treated between December 2009 and January 2014 (n=137) for anterior circulation stroke were divided into age groups. Patient data including National Institutes of Health Stroke Scale (NIHSS) and Alberta Stroke Program Early CT Score (ASPECTS) scoring pre and post procedure and procedural timings were collected. Modified Rankin score was used as functional outcome measure at 90 days.

Results: For the $>70$ age group, ASPECTS correlated most strongly with outcome (ASPECTS $>7$ - OR 3.75, 95\% CI 1.04-13.5) and mortality (ASPECTS $<8$ - OR 5.08, CI 1.1-23.3). In the $<70$ age group, only NIHSS correlated with both outcome (NIHSS $<20-$ OR 4.17, CI 1.4-12.2) and mortality (NIHSS $>20$ - OR 6.22, CI 1.4-26.7) $(\mathrm{p}<0.05)$. Both NIHSS and ASPECTS done at $24 \mathrm{~h}$ were strongly associated with outcome at 90 days. Revascularisation $>5 \mathrm{~h}$ after onset in the $<70$ age group was associated with markedly poorer outcome with increased risk of malignant MCA transformation, while this trend was not seen in the $>70$ age group.
\end{abstract}

Conclusions: ASPECT scoring at admission is clinically helpful in patient selection for thrombectomy in the $>70$ age group as it correlates well with outcome and mortality, but less useful in the $<70$ age group, possibly due to the increased plasticity of remodelling. Revascularisation time appears to be much more important in the younger age group, and restoration of circulation within 5 hours should be the goal to reduce the risk of reperfusion injury.

Highlights

- ASPECTS predicts outcome with greater sensitivity than NIHSS in the $>70$ age group

- In the $<70$ age group, NIHSS was a better predictor and ASPECTS not significant

- Extending times greater than 5 hours in the $<70$ age group risks reperfusion injury

- Post procedure, both NIHSS and ASPECTS both predict 90 day outcome accurately

\section{Introduction}

Mechanical thrombectomy (MT) is an experimental interventional treatment for ischaemic stroke in the UK [1], which has been shown to offer increased rates of recanalisation in cases of large vessel occlusion compared to IV treatment alone or combined IV/IA thrombolysis [2]. Early randomised controlled trials failed to show benefit comparing MT to conventional treatment [3-6], but these trials were criticized for failing to optimise patient selection [7] and used older devices. Recently, four new large randomised controlled trials addressing these issues have proven significant benefit in functional outcome for MT over IV thrombolysis in selected patient groups [8-11].

Alberta Stroke Program Early CT Score (ASPECTS) is a radiological scoring system that places equal weighting on the regions and important structures in the middle cerebral artery territory and can be scored using plain CT, CT-perfusion (CBVASPECTS) and diffusion weighted MRI (MRI-ASPECTS)[12]. The different modes have been shown to yield similar scores, though MRI-ASPECTS tends to score lower; however, many patients have contraindications to MRI on an acute admission $[13,14]$. ASPECTS has been shown in many studies investigating both thrombolysis and endovascular intervention (intra-arterial thrombolysis) to be an independent predictor of outcome, often with a threshold of $<8$ at admission heralding poorer function at 3 months [15-17]. On the other hand, some studies do not agree on the significance of early ischaemic changes on CT as predictors of long term functional outcome [18].

Age has also been shown to be an important factor in recovery after treatment for ischaemic stroke, with age groups $\geq 70-80$ years associated with a significant reduction in recanalization rates [19]. Even when adjusted for recanalization, this age group displayed poorer clinical outcomes, with a trend of better outcomes with decreasing patient age $[20,21]$.

Time to recanalization has been shown to be important in determining outcome. Younger patients presenting with large 
vessel occlusion tend to be treated more aggressively and this may result in guideline breach times being broken. However, it is not currently clear whether this has an effect on patient outcome.

In this study, we evaluate the use of both the clinical National Institutes of Health Stroke Score (NIHSS) and CT ASPECTS in the selection of patients for endovascular thrombectomy and at 24 hours post procedure in evaluating patient prognosis, with further analysis to ascertain whether factors affecting prognosis differ between the $<70$ and $\geq 70$ year age groups. Recanalisation times between groups are also analysed to determine whether younger patients have increased procedural times and whether this results in differences in 90 day outcome.

\section{Methods}

All patients who had received endovascular intervention for anterior circulation stroke at our institution and who were on the thrombectomy register were recruited retrospectively for this study. Intervention was defined as instigating a procedure with intent to progress to mechanical thrombectomy, although this was occasionally not performed if no clot was found or the procedure could not be completed.

Retrospective ASPECT scoring was performed by two neuroradiology trainees on plain CT done at arrival into the Accident and Emergency department and at 24 hours post procedure, as described by Barber et al. [12]. CT examinations were done on a Siemens Somatom Definition AS (Siemens, Erlangen, Germany).

The pathway for neurointervention at our institution has previously been described [22]. Briefly, patients are selected for thrombectomy if they present within 4.5 hours of onset of anterior circulation symptoms and have a treatable carotid bifurcation or proximal middle cerebral artery (M1 or M2) occlusion with no haemorrhagic component or significant established ischaemic change. Age should be $<=80$ years, but exceptions are made on a case by case basis dependant on previous function. NIHSS scoring is done by the on-call stroke team on initial examination. Bridging intravenous therapy with alteplase $0.9 \mathrm{mg} / \mathrm{kg}$ is given if there are no contraindications as a $10 \%$ bolus and $90 \%$ infusion with $30 \%$ dose retained for intra-arterial (IA) use.

Thrombectomy is attempted under general anaesthesia or sedation via femoral arterial access using either a stentbased retrieval device or suction by hand, or by the Penumbra Aspiration Pump® (Penumbra Inc., Alameda, CA, USA) for proximal occlusions. Residual distal thrombus disruption by microwire and IA nimodipine for vasospasm are used if necessary. The procedure is continued until revascularisation is attained, the procedure is abandoned due to technical or patient reasons or the time limit of 6 hours from onset is reached. If transient reperfusion is attained, longer time limits are allowed on a case by case basis.

On completion of the procedure, the femoral arterial catheter is removed and arterial closure device is applied. Blood pressure is maintained at or less than 185/110 and antiplatelet agents and anticoagulants are not routinely used for 24 hours post-procedure unless a stent is placed, in which case $500 \mathrm{mg}$ aspirin IV is given and dual oral antiplatelet therapy instigated. Otherwise, oral antiplatelet agents are started at 24 hours as per UK guidelines if the CT showed no haemorrhage [23]. If minor haemorrhagic transformation is shown, aspirin is started at reduced dose (75mg OD). At 24 hours NIHSS is repeated by the stroke team. $\mathrm{mRS}$ is scored at discharge and at 90 days post procedure. Onset to completion time is defined as the time between symptomatic onset and the timing of the final catheter angiogram.

Differences between groups were investigated by odds ratios and Mann-Whitney U-test (with $\mathrm{p}<0.05$ considered as significant). Independent predictors of outcome were analysed by binary linear regression analysis. SPSS (v.22) was used for all statistical analyses. Cause of death was ascertained for all patients deceased at 90 days.

\section{Results}

\section{Outcomes}

Of a total of 166 patients in the thrombectomy register at the time of the study, 137 were included in this study with anterior circulation strokes, with the remainder being posterior circulation. Of these, $47 \%$ were male and $53 \%$ were female, with age ranging from 20 to 90 years (average 63.2). 82 patients fell into the 20-69 age group and 55 patients were over or equal to 70 years of age. 131 patients had a pre-intervention CT and 135 had a post intervention CT. Thrombectomy was not attempted in 13 patients $(9.5 \%)$ due to it not being required or difficulties during the procedure. Eight patients developed significant postprocedure haemorrhage as determined by SITS-MOST criteria [24] with deterioration of clinical condition (5.8\%). Thirty patients had a contra-indication to intravenous thrombolysis meaning that $77.4 \%$ of the total patient group received bridging therapy.

Total all-cause mortality at 90 days was 20 of 137 (14.5\%) and an outcome of functional independence (mRS 0-2) was achieved in $57 / 137$ patients (41.6\%). This is a better result than earlier clinical trials $[11,25]$, but not as was achieved in the most recently published studies (8-10), as our dataset encompasses patients dating back to 2009. There was observed an increase in mortality and decrease in mRS $0-2$ with age, with only $30 \%$ achieving independence and $30 \%$ mortality in the $>80$ age group.

Mortality cause analysis of the patients deceased at 90 days was undertaken. Twelve deaths in the $<70$ years age group were found (14.6\%), with 2 deaths in the $20-40$ years age group. Of these, 9 were due to malignant MCA transformation of stroke (including the 2 in the 20-40 years group) and 3 of other causes $(75 \%)$. In the $\geq 70$ years group, there were 9 deaths, of which all were due to complications relating to stroke, but only 4 of which had malignant MCA transformation (44.4\%). Malignant MCA was observed in 20 of 137 patients in this study with an average age of 56.9 years. Mortality was $100 \%$ in the $\geq 70$ years group and $56.3 \%$ in the $20-69$ years group (Table 1 ).

\section{NIHSS}

Patients were divided into two age groups: 20-69 years and 
$\geq 70$ years for the remaining analyses. NIHSS was highly predictive of outcome independent of age, with a pre-procedure score of $>20$ (severe stroke) associated with a significant drop in favourable outcome and increase in mortality compared with the next group (moderate to severe, 16-20, Figure 1). Independent outcome dropped from $51.2 \%$ to $23.4 \%$, with mortality rising from $12.2 \%$ to $25.5 \%$. NIHSS at $24 \mathrm{~h}$ post-procedure was predictably more accurate, with a score of $<16$ associated with $74.2 \%$ probability of favourable outcome and $3.0 \%$ risk of death, changing to $5 \%$ and $40 \%$ respectively in the $>20$ group.

\section{ASPECTS}

ASPECT scores were then divided into three groups: 0-4, 5-7 and 8-10, with 10 representing no ischaemic change and 0 complete infarction of the MCA territory. There was an increase in favourable outcome with increasing ASPECT score group in both age groups to a maximum of $50 \%$ in the $8-10$ group (Figure 2). Mortality decreased with ASPECT score, but again there was an unexpected excess mortality in the 8-10 group for the 20-69 years age group.

At 24 hours, ASPECTS groupings showed increased correlation with outcome and mortality, especially in the $\geq 70$ years age group with $80 \%$ of patients with ASPECTS of 8 or greater achieving independence compared to $50 \%$ in the $5-7$ group. A score of 0-4 was strongly linked with mortality, with one third (33.3\%) mortality in the $\geq 70$ years group compared to $4.5 \%$ in the 5-7 group (Figure 3).

\section{Non-parametric analyses}

Non-parametric comparison of absolute (non-categorised)

Table 1: Malignant MCA (MMCA) relation with mortality in the $20-69$ and $\geq 70$ age groups.

\begin{tabular}{|c|c|c|c|}
\hline & MMCA Prevalence & MMCA Mortality & $\begin{array}{l}\text { MMCA mortality/All cause } \\
\text { mortality }\end{array}$ \\
\hline Total age groups & $20 / 137$ (14.6\%) & $13 / 20(65 \%)$ & $13 / 21(61.9 \%)$ \\
\hline Age $\geq 70$ & $4 / 55(7.2 \%)$ & $4 / 4(100 \%)$ & $4 / 9(44.4 \%)$ \\
\hline
\end{tabular}
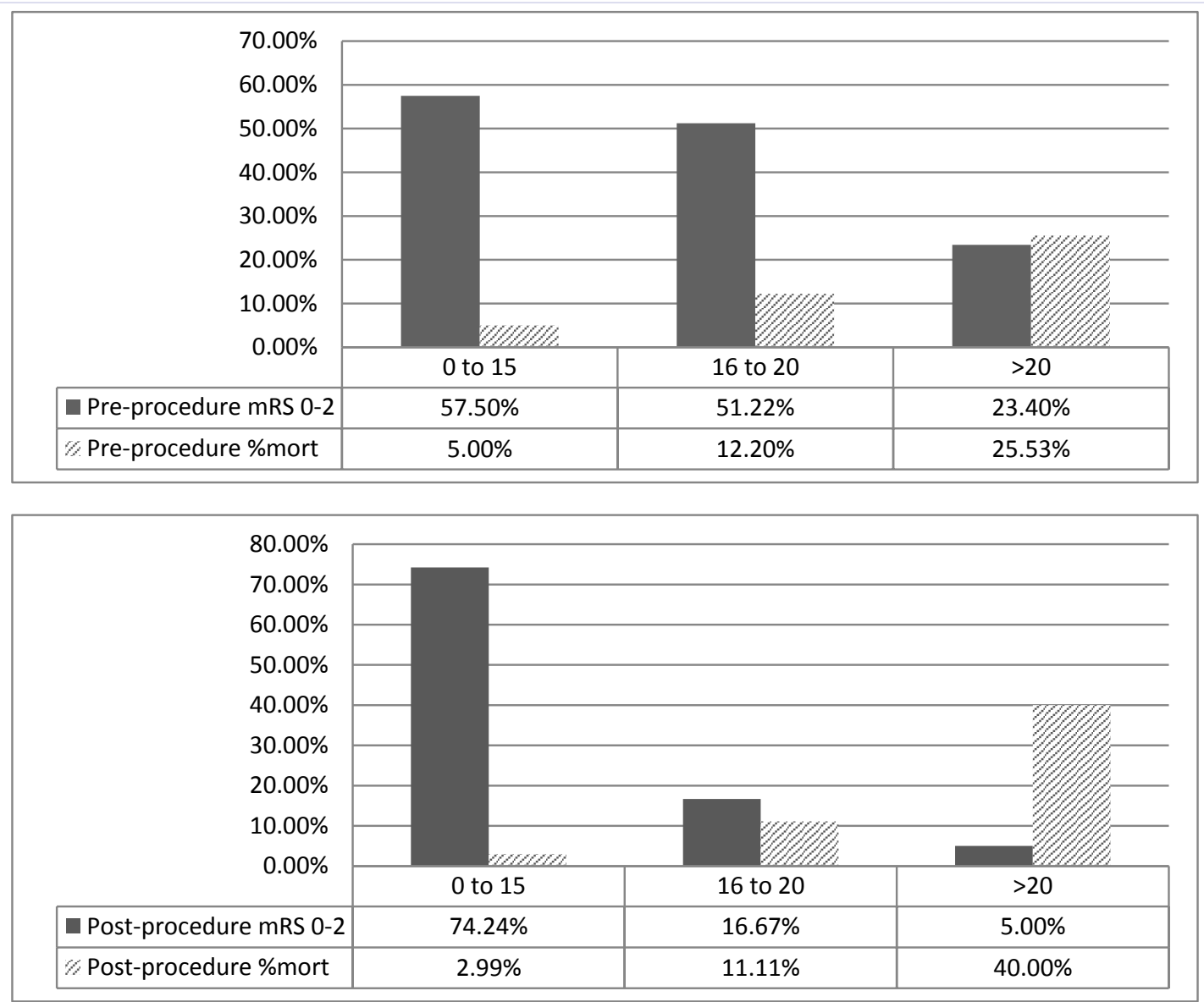

Figure 1: Pre-procedural (top) and post-procedural (bottom) NIHSS related to outcome (expressed as mRS at 90 days) and mortality (mRS 6 at 90 days). 

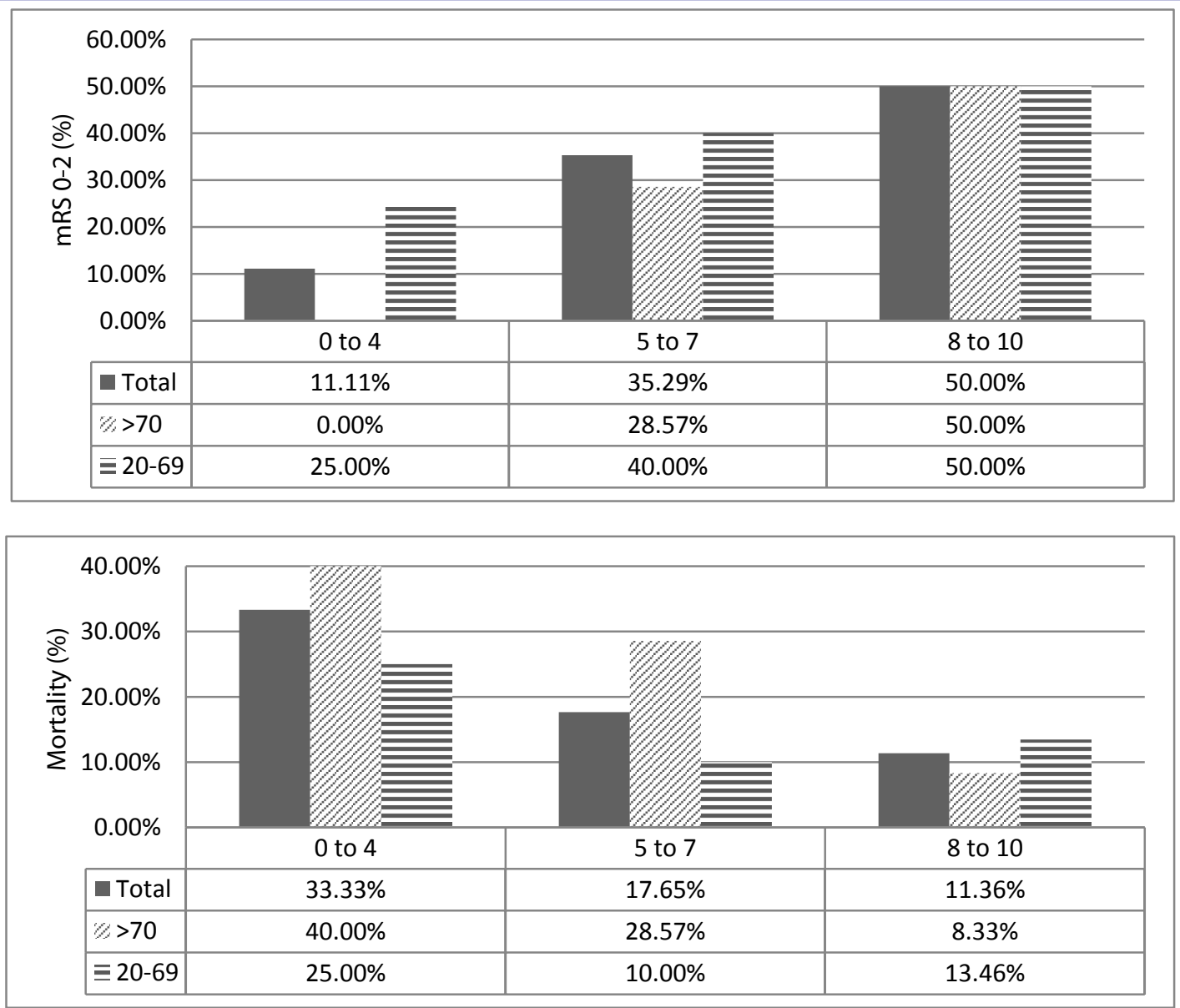

Figure 2: Pre-procedural ASPECTS related to outcome (expressed as mRS at 90 days, top) and mortality (mRS 6 at 90 days, bottom.

pre-intervention scores between outcome groups within the two age ranges showed significant differences in median ASPECT score between good/poor functional outcome groups and for mortality in the $\geq 70$ years age group $(\mathrm{p}<0.05)$. NIHSS was significant for functional outcome only in this age range. For the 20-69 years age range, both functional outcome and mortality showed significant differences in median NIHSS $(p<0.05)$, with ASPECTS showing no significant difference between groups.

\section{Pre-procedural scoring evaluation}

The most important predictive score differed between age groups. In the $\geq 70$ years group, the most important preprocedure score for independent outcome (mRS 0-2) was ASPECTS of $>7$ (OR 3.75, 95\% CI 1.04-13.5) (Sensitivity 81.8\%, specificity $45.4 \%$ (sens/spec) for ASPECTS $>7$ compared to $77.2 \%$ and $56.7 \%$ for NIHSS $<20$ ). For mortality in this group, the most important score was ASPECTS of $<8$ (OR 5.08, CI 1.123.3) and NIHSS was not significant (sens/spec 66.7\%/71.7\%). However, for the 20-69 years age group, ASPECTS was not significant and the most important score for outcome was NIHSS (sens/spec 56.9\%/76.0\% for NIHSS $<20$, ASPECTS $>7$ not significant). NIHSS $<20$ was associated with 4.17 times greater chance of independent outcome (CI 1.4-12.2) and NIHSS of $>20$ was associated with 6.22 times greater risk of death (CI 1.4-26.7) (sens/spec 70.0\%/72.7\%).

\section{Post-procedural scoring evaluation}

At $24 \mathrm{~h}$ post-procedure, NIHSS was the greater predictor of outcome in both groups, with NIHSS $<15$ associated with 28.9 times greater probability of independence at 90 days (CI 10.0-83.6). NIHSS cutoff of 15 compared to 20 conferred a slight reduction in sensitivity, but a significant increase in specificity (sens/spec 96.2\%/55.0\% compared to 90.7\%/76.8\% for combined age groups). For mortality, ASPECTS was more important in the $\geq 70$ years age group, with score of $<5$ conferring a 15.5 times greater risk of death (CI 1.74-138) (sens/spec 87.5\%/68.9\%). Both ASPECTS and NIHSS were important in the 20-69 years age group and combined ASPECTS of $<5$ with NIHSS $>20$ conferred 40 times greater risk (CI 7.24-221) (sens/spec $83.3 \% / 88.9 \%)$

\section{Linear regression modelling}

Further binary linear regression modelling was attempted with age, absolute (non-categorised) ASPECTS and NIHSS scores as variables determining functional outcome. Insufficient data was available to model mortality. Pre-intervention, NIHSS 
was the only independent variable when both age groups are considered ( $\mathrm{p}=0.01$ for 20-69 group and combined groups), but not significant in the $\geq 70$ years group when considered separately $(\mathrm{p}=0.07)$. ASPECTS was not an independent variable for either age group. Post intervention, ASPECTS and NIHSS were both significant independent variables for functional outcome $(\mathrm{p}<0.05)$.

\section{Onset to completion timing}

Analysis of onset to completion time figures showed that there were more breaches of the 6 hour limit in the 20-69 years group compared to the $\geq 70$ years group $(35 / 78,43.7 \%$, compared to $16 / 52,30.7 \%$ ). There was no significant difference between breaching between the youngest patients in the 20-40 group and 41-69 group when considered separately (43.2\% and $46.2 \%$ respectively), although more patients in the $20-40$ years group breached 7 hours (38.6\% compared to $13.4 \%$ and $9.6 \%$ in the 40-69 years group and $\geq 70$ years group respectively). Correlating onset to completion time with outcome showed significant increase in mortality and infirmity over 5 hours in both age groups, but there was also a marked increase in mortality above 5 hours in the 20-69 years group. Of the 27 patients in this group revascularised in under 5 hours there was 1 death $(3.7 \%)$, rising to $22.2 \%$ in the $5-6 \mathrm{~h}$ period $(4 / 18)$ and $>6 \mathrm{~h}$ group $(7 / 33)$. In the $\geq 70$ years group, the trend was less marked, with values of $9.5 \%(2 / 20), 12.5 \%(2 / 16)$ and $18.8 \%(3 / 16)$ for the same time periods, respectively. Two patients had no time data available. Looking at the onset to completion times for the patients developing malignant MCA specifically, it was shown that only 1 case occurred before $5 \mathrm{~h}(1 / 48,2.1 \%)$. Between 5-6 hours there were 7 cases $(7 / 34,20.6 \%)$ and over 6 hours there were 10 cases $(10 / 53,18.9 \%)$. This equated to a sensitivity of $100 \%$ /specificity $41.5 \%$ for malignant MCA in the 20-69 age group for an onset to completion time of more than $5 \mathrm{~h}$.

\section{Discussion}

In this study we have shown that the prognostic value of pre-procedural scoring is dependent on patient age, with clinical scoring (NIHSS) having greater value in younger patients and radiological scoring (ASPECTS) having more significance in older patients. We have also shown that younger patients appear more sensitive to recanalization timing, with longer onset to completion time reflected in worse outcomes and higher mortality with increased risk of malignant MCA.

Age is generally considered to be an important factor in determining prognosis after stroke intervention. Several studies show increasing age correlation with unfavourable outcomes [21,26,27] with lower rates of recanalization [19] and higher mortality and haemorrhage [28], especially in the $>80$ age group. Older patients also demonstrated a higher rate

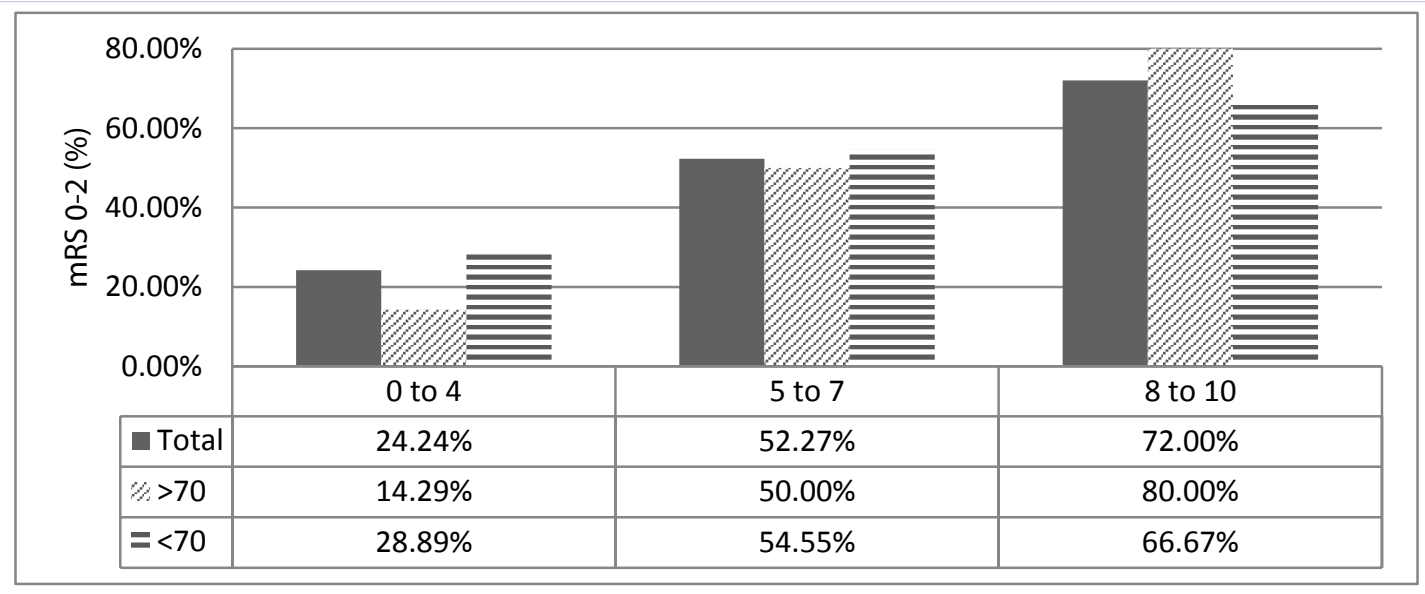

\begin{tabular}{|c|c|c|c|}
\hline \multirow{4}{*}{ 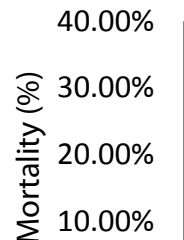 } & & & \\
\hline & \multicolumn{3}{|l|}{$\mathscr{W} \mathbb{Z} \mathbb{Z}$} \\
\hline & \multicolumn{3}{|c|}{ 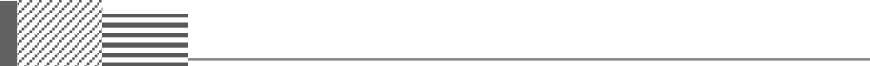 } \\
\hline & & & \\
\hline \multirow{2}{*}{$0.00 \%$} & & $\mathscr{W} \mathbb{W}$ & \\
\hline & 0 to 4 & 5 to 7 & 8 to 10 \\
\hline - Total & $28.79 \%$ & $2.27 \%$ & $0.00 \%$ \\
\hline$\not / Z>70$ & $33.33 \%$ & $4.55 \%$ & $0.00 \%$ \\
\hline$=<70$ & $26.67 \%$ & $0.00 \%$ & $0.00 \%$ \\
\hline
\end{tabular}

Figure 3: Pre-procedural ASPECTS related to outcome (expressed as mRS at 90 days, top) and mortality (mRS 6 at 90 days, bottom). 
of futile recanalization $[20,21]$ (poor outcome despite good flow outcomes).

A variety of studies have shown that NIHSS is an independent predictor of outcome at 90 days for both intra-arterial thrombolysis (IAT) and thrombectomy [29-31]. It has also been shown that higher NIHSS is associated with higher rates of futile recanalization [20] and haemorrhage [32]. However, there is some division over the utility of ASPECTS in the pre-intervention stage in predicting 90 day outcome and mortality. A number of papers show that a higher ASPECTS of $>7$ is associated with improved outcomes in intra-arterial thrombolysis [15-17,33], with lower scores associated with poorer recanalisation and increased risk of haemorrhage. However, none directly compare younger with older patient age groups, although one study using IAT investigated ASPECTS specifically in the elderly ( $>80$ years age group, [34]).

In studies using mechanical thrombectomy, three studies $[26,29,35]$ also demonstrate an association of ASPECTS with outcome, but two did not demonstrate this association $[18,30]$. One study comparing the CT ASPECTS and CT perfusion ASPECTS found that only $\triangle(\mathrm{CBV}-\mathrm{CBF})$ ASPECTS was a significant predictor of successful recanalization [36], with plain CT ASPECTS having a sensitivity of only $77 \%$ and specificity $42 \%$ for favourable outcome. Again none of the above studies analysed outcome data by age group.

In our study, we showed that ASPECTS was not significantly correlated with outcome in the 20-69 years age group, with NIHSS of 20 the most significant cut-off for favourable outcome or mortality. However, in the $\geq 70$ group, ASPECTS scores were significantly different between outcome groups and an ASPECTS cut off of 8 was the most important factor for prognosis, while NIHSS was less important. In linear regression models, NIHSS was the only important independent variable, but was not significant in the $\geq 70$ group when considered separately. At 24 hours postprocedure, both ASPECTS and NIHSS are independent predictors of outcome, with ASPECTS more important for predicting mortality in both age groups and NIHSS more important for functional outcome.

Short time from onset to recanalization is generally accepted to be an important prognostic factor, as there is less neuronal damage and associated risk of oedematous changes such as malignant MCA. It has been shown to be associated with improved outcome [37]. In our institution, we currently use a 4.5 hour cutoff from onset for instigation of intervention to complete within 6 hours. However, in our data we can see that there is a dramatic reduction in favourable outcome and increase in mortality above 5 hours from onset, which is especially marked in the younger age group which demonstrated an increase from $3.7 \%$ to $22.2 \%$ mortality comparing $<5 \mathrm{~h}$ to $5-6 \mathrm{~h}$ onset to completion times. We also found an increase in incidence of malignant MCA syndrome, with 17 of 18 malignant MCA syndromes occurring with onset to completion times above 5 hours. It was also observed that of the 16 malignant MCAs in the 20-69 years age group, 10 presented with NIHSS $>20$, indicating a large vessel occlusion (of which 7 of the 9 deaths also had NIHSS $>20$ ). It is known that malignant
MCA syndrome is more common in the younger age group [38], likely due to increased inflammatory response and less space in the skull vault for oedematous expansion. We may therefore surmise that increased mortality in the younger age group may have been caused by increased procedural times leading to later revascularisation in patients with large vessel occlusions resulting in reperfusion damage and malignant MCA.

\section{Conclusion}

In this study we have shown that both clinical NIHSS and radiological ASPECTS are important in predicting outcome in patients undergoing mechanical thrombectomy. In younger patients under the age of 70, ASPECTS correlates poorly with outcome and mortality and NIHSS is more significant. However, it is of utility in the $\geq 70$ years age group, where it correlates well with both functional outcome and mortality together with NIHSS. Post-procedurally, both NIHSS and ASPECTS are helpful in predicting the clinical course of recovery regardless of age group. We also showed increased infirmity and mortality rates in the younger age group, likely due to reperfusion injury when onset to completion times extended above 5 hours (compared to the currently used 6 hour cutoff). In younger patients presenting late or at the borderline, it would be prudent to weigh the benefits and risks of undertaking thrombectomy and risking reperfusion injury compared to letting the infarct take its course with best supportive care with or without thrombolysis. The data in this study supports reducing the recommended onset to completion time to 5 or 5.5 hours from 6 hours for this younger age group, especially if patients present with NIHSS greater than 20 .

\section{Competing interests}

No grants were obtained for undertaking this study. The authors declare that there are no competing interests.

\section{References}

1. Mechanical clot retrieval for treating acute ischaemic stroke | Guidance and guidelines | NICE n.d. https://www.nice.org.uk/guidance/ipg458 (accessed June 30, 2015).

2. Rha J-H, Saver JL. The impact of recanalization on ischemic stroke outcome: a meta-analysis. Stroke. 2007;38:17272772. doi:10.1161/01. STR.0000258112.14918.24.

3. Broderick JP, Palesch YY, Demchuk AM, Yeatts SD, Khatri P, Hill $\mathrm{MD}$, et al. Endovascular therapy after intravenous t-PA versus t-PA alone for stroke. N Engl J Med. 2013;368:23390923. doi:10.1056/ NEJMoa1214300.

4. Kidwell CS, Jahan R, Gornbein J, Alger JR, Nenov V, Ajani Z, et al. A Trial of Imaging Selection and Endovascular Treatment for Ischemic Stroke. New England Journal of Medicine. 2013;368:23394476. doi:10.1056/ NEJMoa1212793.

5. Singh P, Kaur R, Kaur A. Endovascular treatment of acute ischemic stroke. J Neurosci Rural Pract. 2013;4:24250163. doi:10.4103/09763147.118787.

6. Smith WS, Sung G, Saver J, Budzik R, Duckwiler G, Liebeskind DS, et al. Mechanical thrombectomy for acute ischemic stroke: final results of the Multi MERCI trial. Stroke. 2008;39:18309168. doi:10.1161/ STROKEAHA.107.497115.

7. Qureshi AI, Abd-Allah F, Aleu A, Connors JJ, Hanel RA, Hassan AE, 
et al. Endovascular treatment for acute ischemic stroke patients: implications and interpretation of IMS III, MR RESCUE, and SYNTHESIS EXPANSION trials: A report from the Working Group of International Congress of Interventional Neurology. J Vasc Interv Neurol. 2014;7:24920991.

8. Saver JL, Goyal M, Bonafe A, Diener H-C, Levy EI, Pereira VM, et al. Stent-retriever thrombectomy after intravenous t-PA vs. t-PA alone in stroke. N Engl J Med. 2015;372:25882376. doi:10.1056/ NEJMoa1415061.

9. Goyal M, Demchuk AM, Menon BK, Eesa M, Rempel JL, Thornton J, et al. Randomized assessment of rapid endovascular treatment of ischemic stroke. N Engl J Med. 2015;372:25671798. doi:10.1056/ NEJMoa1414905.

10. Campbell BCV, Mitchell PJ, Kleinig TJ, Dewey HM, Churilov L, Yassi $\mathrm{N}$, et al. Endovascular therapy for ischemic stroke with perfusionimaging selection. N Engl J Med. 2015;372:25671797. doi:10.1056/ NEJMoa1414792.

11. Berkhemer OA, Fransen PSS, Beumer D, van den Berg LA, Lingsma HF, Yoo AJ, et al. A Randomized Trial of Intraarterial Treatment for Acute Ischemic Stroke. New England Journal of Medicine. 2015;372:25517348. doi:10.1056/NEJMoa1411587.

12. Barber PA, Demchuk AM, Zhang J, Buchan AM. Validity and reliability of a quantitative computed tomography score in predicting outcome of hyperacute stroke before thrombolytic therapy. ASPECTS Study Group. Alberta Stroke Programme Early CT Score. Lancet. 2000;355:10905241.

13. Nezu T, Koga M, Nakagawara J, Shiokawa Y, Yamagami H, Furui E, et al. Early ischemic change on CT versus diffusion-weighted imaging for patients with stroke receiving intravenous recombinant tissue-type plasminogen activator therapy: stroke acute management with urgent risk-factor assessment and improvement (SAMURAI) rt-PA registry. Stroke. 2011;42:21719764. doi:10.1161/STROKEAHA.111.614404.

14. Barber P, Hill M, Eliasziw M, Demchuk A, Pexman J, Hudon M, et al. Imaging of the brain in acute ischaemic stroke: comparison of computed tomography and magnetic resonance diffusion-weighted imaging. J Neurol Neurosurg Psychiatry. 2005;76:16227545. doi:10.1136/jnnp.2004.059261.

15. Nezu T, Koga M, Kimura K, Shiokawa Y, Nakagawara J, Furui E, et al. Pretreatment ASPECTS on DWI predicts 3-month outcome following rt-PA: SAMURAI rt-PA Registry. Neurology. 2010;75:20697108. doi:10.1212/WNL.0b013e3181eccf78.

16. Hill MD, Rowley HA, Adler F, Eliasziw M, Furlan A, Higashida RT, et al. Selection of acute ischemic stroke patients for intra-arterial thrombolysis with pro-urokinase by using ASPECTS. Stroke. 2003;34:12843342. doi:10.1161/01.STR.0000082483.37127.D0.

17. Tsivgoulis G, Saqqur M, Sharma VK, Lao AY, Hoover SL, Alexandrov AV, et al. Association of pretreatment ASPECTS scores with tPAinduced arterial recanalization in acute middle cerebral artery occlusion. J Neuroimaging. 2008;18:18190497. doi:10.1111/j.15526569.2007.00169.x.

18. Patel SC, Levine SR, Tilley BC, Grotta JC, Lu M, Frankel M, et al. Lack of clinical significance of early ischemic changes on computed tomography in acute stroke. JAMA. 2001;286:11735758.

19. Qureshi AI, Suri MFK, Georgiadis AL, Vazquez G, Janjua NA. IntraArterial Recanalization Techniques for Patients 80 Years or Older with Acute Ischemic Stroke: Pooled Analysis from 4 Prospective Studies. AJNR Am J Neuroradiol. 2009;30:19342542. doi:10.3174/ajnr.A1503.
20. Hussein HM, Georgiadis AL, Vazquez G, Miley JT, Memon MZ, Mohammad YM, et al. Occurrence and predictors of futile recanalization following endovascular treatment among patients with acute ischemic stroke: a multicenter study. AJNR Am J Neuroradiol. 2010;31:20075087. doi:10.3174/ajnr.A2006.

21. Singer OC, Haring H-P, Trenkler J, Nolte CH, Bohner G, Reich A, et al. Age dependency of successful recanalization in anterior circulation stroke: the ENDOSTROKE study. Cerebrovasc Dis. 2013;36:24281318. doi:10.1159/000356213.

22. Ahmad N, Nayak S, Jadun C, Natarajan I, Jain P, Roffe C. Mechanical thrombectomy for ischaemic stroke: the first UK case series. PLoS ONE. 2013;8:24386090. doi:10.1371/journal.pone.0082218.

23.SINAP (Stroke Improvement National Audit Programme) | Royal College of Physicians n.d. https://www.rcplondon.ac.uk/projects/ stroke-improvement-national-audit-programme-sinap (accessed June 30, 2015).

24. Wahlgren N, Ahmed N, Eriksson N, Aichner F, Bluhmki E, Dávalos A, et al. Multivariable analysis of outcome predictors and adjustment of main outcome results to baseline data profile in randomized controlled trials: Safe Implementation of Thrombolysis in StrokeMOnitoring STudy (SITS-MOST). Stroke. 2008;39:18927461. doi:10.1161/STROKEAHA.107.510768.

25. Smith WS, Sung G, Saver J, Budzik R, Duckwiler G, Liebeskind DS, et al. Mechanical thrombectomy for acute ischemic stroke: final results of the Multi MERCI trial. Stroke. 2008;39:18309168. doi:10.1161/ STROKEAHA.107.497115.

26. Almekhlafi MA, Davalos A, Bonafe A, Chapot R, Gralla J, Pereira VM, et al. Impact of age and baseline NIHSS scores on clinical outcomes in the mechanical thrombectomy using solitaire FR in acute ischemic stroke study. AJNR Am J Neuroradiol. 2014;35:24557701. doi:10.3174/ajnr. A3855.

27. Rai AT, Jhadhav Y, Domico J, Hobbs GR. Procedural predictors of outcome in patients undergoing endovascular therapy for acute ischemic stroke. Cardiovasc Intervent Radiol. 2012;35:22167306. doi:10.1007/s00270-011-0323-7.

28. Duffis EJ, He W, Prestigiacomo CJ, Gandhi CD. Endovascular treatment for acute ischemic stroke in octogenarians compared with younger patients: a meta-analysis. Int J Stroke. 2014;9:23981475. doi:10.1111/ ijs.12098.

29. Kurre W, Aguilar-Pérez M, Niehaus L, Fischer S, Schmid E, Bäzner $\mathrm{H}$, et al. Predictors of outcome after mechanical thrombectomy for anterior circulation large vessel occlusion in patients aged $=80$ years. Cerebrovasc Dis. 2013;36:24281266. doi:10.1159/000356186.

30. Costalat V, Lobotesis K, Machi P, Mourand I, Maldonado I, Heroum $\mathrm{C}$, et al. Prognostic factors related to clinical outcome following thrombectomy in ischemic stroke (RECOST study). 50 patients prospective study. Eur J Radiol. 2012;81:22940230. doi:10.1016/j. ejrad.2012.07.012.

31. Molina CA, Alexandrov AV, Demchuk AM, Saqqur M, Uchino $\mathrm{K}$, Alvarez-Sabín J, et al. Improving the predictive accuracy of recanalization on stroke outcome in patients treated with tissue plasminogen activator. Stroke. 2004;35:14671245. doi:10.1161/01. STR.0000106485.04500.4A.

32. Kuntze Söderqvist A, Kaijser M, Söderman M, Holmin S, Wahlgren $\mathrm{N}$, Andersson T. Mechanical thrombectomy in acute ischemic stroke-experience from 6 years of practice. Neuroradiology. 2014;56:24687568. doi:10.1007/s00234-014-1353-z. 
33. Hill MD, Demchuk AM, Goyal M, Jovin TG, Foster LD, Tomsick TA, et al. Alberta Stroke Program early computed tomography score to select patients for endovascular treatment: Interventional Management of Stroke (IMS)-III Trial. Stroke. 2014;45:24335227. doi:10.1161/ STROKEAHA.113.003580.

34. Sylaja PN, Cote R, Buchan AM, Hill MD, Canadian Alteplase for Stroke Effectiveness Study (CASES) Investigators. Thrombolysis in patients older than 80 years with acute ischaemic stroke: Canadian Alteplase for Stroke Effectiveness Study. J Neurol Neurosurg Psychiatr. 2006;77:16505004. doi:10.1136/jnnp.2005.086595.

35. Soize S, Barbe C, Kadziolka K, Estrade L, Serre I, Pierot L. Predictive factors of outcome and hemorrhage after acute ischemic stroke treated by mechanical thrombectomy with a stent-retriever. Neuroradiology. 2013;55:23644538. doi:10.1007/s00234-013-1191-4.
36. Psychogios M-N, Schramm P, Frölich AM, Kallenberg K, Wasser K, Reinhardt L, et al. Alberta Stroke Program Early CT Scale evaluation of multimodal computed tomography in predicting clinical outcomes of stroke patients treated with aspiration thrombectomy. Stroke. 2013;44:23715960. doi:10.1161/STROKEAHA.113.001068.

37. Raoult $\mathrm{H}$, Eugène $\mathrm{F}$, Ferré $\mathrm{J}-\mathrm{C}$, Gentric $\mathrm{J}$-C, Ronzière $\mathrm{T}$, Stamm $\mathrm{A}$, et al. Prognostic factors for outcomes after mechanical thrombectomy with solitaire stent. J Neuroradiol. 2013;40:23684343. doi:10.1016/j. neurad.2013.04.001.

38. Oppenheim C, Samson Y, Manaï R, Lalam T, Vandamme X, Crozier S, et al. Prediction of malignant middle cerebral artery infarction by diffusion-weighted imaging. Stroke. 2000;31:10978048. 